\title{
ERRATA
}

\section{Erratum to: Synthesis and Antimicrobial Activity of New Dialkyl(diaryl)-2-(5-chloro-2-hydroxyphenyl)- 2-(phenylethenyl)-pentylphosphonium Salts}

\author{
D. A. Tatarinov ${ }^{a, b}$, N. V. Terekhova ${ }^{a, b}$, A. D. Voloshina ${ }^{a}$, \\ A. S. Sapunova ${ }^{a}$, A. P. Lyubina ${ }^{a, b}$, and V. F. Mironov ${ }^{a, b}$ \\ ${ }^{a}$ Arbuzov Institute of Organic and Physical Chemistry, Kazan Scientific Center of the Russian Academy of Sciences, \\ ul. Akademika Arbuzova 8, Kazan, Tatarstan, 420088 Russia \\ *e-mail:datint@iopc.ru \\ ${ }^{b}$ Kazan (Volga Region) Federal University, Kazan, Tatarstan, Russia
}

Received March 15, 2019

DOI: $10.1134 / \mathrm{S} 1070363219030344$

The row in the table on p. 1802 for compound 8a should be as follows:

\begin{tabular}{l|c|c|c|c|c|c|c|c}
\hline \multirow{2}{*}{ Compound } & \multicolumn{2}{|c|}{ Staphylococcus aureus } & \multicolumn{2}{c|}{ Bacillus cereus } & \multicolumn{2}{c|}{$\begin{array}{c}\text { Trichophyton } \\
\text { mentagrophytes }\end{array}$} & \multicolumn{2}{c}{ Candida albicans } \\
\cline { 2 - 8 } & $\begin{array}{c}\mathrm{MIC} \\
\mu \mathrm{g} / \mathrm{mL}\end{array}$ & $\begin{array}{c}\mathrm{MBC}, \\
\mu \mathrm{g} / \mathrm{mL}\end{array}$ & $\begin{array}{c}\mathrm{MIC}, \\
\mu \mathrm{g} / \mathrm{mL}\end{array}$ & $\begin{array}{c}\mathrm{MBC}, \\
\mu \mathrm{g} / \mathrm{mL}\end{array}$ & $\begin{array}{c}\mathrm{MIC}, \\
\mu \mathrm{g} / \mathrm{mL}\end{array}$ & $\begin{array}{c}\mathrm{MFC}, \\
\mu \mathrm{g} / \mathrm{mL}\end{array}$ & $\begin{array}{c}\mathrm{MIC}, \\
\mu \mathrm{g} / \mathrm{mL}\end{array}$ & $\begin{array}{c}\mathrm{MFC}, \\
\mu \mathrm{g} / \mathrm{mL}\end{array}$ \\
\hline $\mathbf{8 a}$ & $125 \pm 10$ & $125 \pm 11$ & $125 \pm 11$ & $125 \pm 12$ & $-^{\mathrm{a}}$ & - & - & - \\
\hline
\end{tabular}

The original article can be found online at https://doi.org/10.1134/S1070363218090062. 